\title{
OBJETIVOS OPERACIONAIS E EFICIENCIA DA APRENDIZAGEM
}

\author{
Joào Alvécio Sossai :
}

\begin{abstract}
RSPU-B/348
Sossal, J. A. Objetivos operacionais e eficiência da aprendizagem. Rev. Saúde públ. S. Paulo, 11: 157-69, 1977.

Resuno: Foi verificado em que medida a aprendizagem pode ser significativamente influenciada pela informação aos estudantes antes do ensino, sobre o que é esperado deles como resultado do ensino. Os sujeitos que participaram deste estudo eram alunos de 4 classes de $5^{a}$ série do 19 Gratl. Dos 140 sujeitos, 69 de duas classes constituiram o grtupo experimental (GE) e 71 de duas outras classes constituiram o grupo de controle $(G C)$. Os sujeitos do $G E$ receberam objetivos operacionais redigidos claramente enquanto que os do $G C$ náo receberam nenhuma informaça sobre objetivos. Foram comparados os GE e GC como um todo, os $G E$ e $G C$ de nivel socio-econômico alto e baixo, os $G E$ e $G C$ com notas altas e baixas, levando em conta, de um lado, mudanças em conhecimentos, atitudes e práticas e, de outtro, mudanças apenas em conhecimentos. Embora os $G E$ apresentaram consistentemente maior aprendizagem do que os $G C$, os resultados nāo foram conclusivos. Quando se comparou os diferentes grupos, observou-se que diferenças significativas na aprendizagem ocorreram em alguns, mas distribuidas ao acaso.
\end{abstract}

UNITERMos: Ensino, objettvos educacionais. Ensino, objetivos operacionais. Aprendizagem.

\section{N T R O D U C A O}

A determinação daquilo que se pretende com a educação é um problema que sempre interessou os responsáveis pela orientação dos mais jovens. Enquanto esta questão estava circunscrita à esfera do lar e da família, não apresentava maiores preocupações para os diversos grupos sociais como ocorre em grande parte até hoje. À medida que parte considerável do papel educativo da família e dos grupos de referência primários foi sendo transferida para $o$ Estado ou para as organizaçōes formais (escolas), a determinação dos objetivos da educação passou a ser preocupação de filósofos e educadores. Aquilo que era preten- dido pela Escola em relação a seus alunos passou a ter relação com o destino cie um pais ou nação.

Com o desenvolvimento da tecnologia e com a crescente modificação de tuda a estrutura social, novos valores surgiram exigindo mudanças sucessivas do sistema de ensino dos diversos paises. Enquanto os ideais da educação medieval eram volíados para valores humanistas, de ordem filosjfica e religiosa, uma sociedade tecnocrata preocupa-se com a formação do homem eficiente, que produza o máxino com o mínimo de esforço. Enquanto os ideais humanisticos se constituiam em algo difícil de ser defi-

* Do Departamento de Prática de Saúde Pública da Faculdade de Saúde Pública da UsP Ar. Dr. Arnaldo, 715 - São Paulo, SP - Brasil. 
SOSSAI, J. A. - Objetivos operacionais e eficiência da aprendizagem. Rev. Saúde públ., S. Paulo, 11: $157-69,1977$

nido, a adaptação ao mundo das máquinas refere-se a coisas exatas e mensuráveis.

Diante desta revolução drástica na sociedade e que se evidenciou principalmente na segunda metade deste século, modificações profundas tiveram que ser introduzidas nas organizações educacionais tendo em vista as novas exigências de uma sociedade altamente industrializada.

Como não poderia deixar de ser, o início desse processo de modificação deveria se iniciar pela redefinição dos objetivos da educação. A preocupação agora é a de educar o homem para um mundo onde predominam a mecanização e a automação, para um mundo que exige alto nivel de especialização como condição para uma adaptação razoável. Nestas circunstâncias $o$ indivíduo deve preparar-se para funcionar como uma peça dentro de um sistema, onde a precisão e a eficiência são características fundamentais.

Este novo esquema, no qual deve estar incluído o homem, exige, portanto, que as agências educacionais encontrem uma nova forma de determinar seus objetivos, de maneira a ser possível especificar, com clareza e precisão, os comportamentos desejáveis ao final de um período em que 0 indivíduo for submetido a um conjunto de estímulos previamente planejados.

Novas estratégias de ensino derivadas de tecnologia do ensino têm demonstrado a eficácia de se determinar de antemão os comportamentos esperados após uma sessão de ensino ou conjunto de sessões. Diversas razões justificam a maior eficiência de um programa de ensino planejado em função de objetivos claramente definidos. Entre elas, pode-se citar a possibilidade de seleção de estratégias adequadas à consecução dos objetivos e de organização de sequiências ótimas do conteúdo de ensino.

Uma questão, entretanto, que parece de capital importância para o ensino $e$ a aprendizagem, não tem recebido a devida atenção dos pesquisadores. Esta questão refere-se à possibilidade de se conseguir maior eficiência no processo de modificação do comportamento, informando aos individuos sobre os objetivos pretendidos pelo instrutor, antes de submetê-los às situações estimuladoras especificas, tendo em vista conseguir as mudanças esperadas.

O presente trabalho tem por finalidade primordial o estudo deste problema cuja importância para a educaçăo em geral e para a educação em saúde, em especial, será discutida oportunamente.

\section{TER MINOLOGIA}

Os diversos especialistas que desenvolveram trabalhos relacionados à determinação de objetivos educativos utilizam uma terminologia variada que, com freqüência, provoca discussões desprovidas de sentido. Essa terminologia inclui as mais variadas expressões para indicar o que chamaremos de "objetivos educativos". Entre os termos ou expressões utilizados podemos citar: fins educacionais, metas educacionais, objetivos gerais, objetivos específicos, objetivos instrucionais, objetivos operacionais.

Com base na literatura tradicional podese afirmar que, na determinação dos objetivos da educação, se procurava distinguir dois tipos: os objetivos a longo prazo e os objetivos a curto prazo. Os primeiros eram chamados de "fins ou metas educacionais" e os segundos simplesmente de "objetivos educacionais". Outra nomenclatura que é encontrada com freqüência para indicar objetivos a longo e a curto prazo é a de chamar os objetivos a longo prazo de "gerais" e os a curto prazo de "especificos"

Trabalhos mais recentes introduzem os termos "instrucional" e "operacional" para indicar objetivos a curto prazo e ao mesmo tempo redigidos de maneira clara e precisa, expressando comportamentos observáveis.

Atualmente tem-se definido objetivo educativo como o comportamento esperado de um individuo ou grupo após submetê-lo a uma situa ça $^{a}$ de ensino-aprendizagem. 
SOSSAI, J. A. - Objetivos operacionais e eficiência da aprendizagem. Rev. Saúde públ., S. Paulo, 11: $157-69,1977$

Os objetivos educativos podem expressar de maneira mais ou menos genérica um comportamento; podem ser, portanto, mais gerais ou mais específicos. Não há um critério absoluto para se determinar o nivel de generalidade ou de especificidade de um objetivo. Um objetivo específico é específico em relação a outro mais genérico que é chamado de geral. Por outro lado, este mesmo objetivo geral pode ser específico em relação a outro.

A relação entre objetivos gerais e especificos só pode ser observada quando comparamos os objetivos de um determinado programa ou quando comparamos objetivos do mesmo programa proposto em vários níveis, tais como nacional, estadual e municipal.

Os termos "instrucional" e "educacional" se equivalem: o termo "instrucional" é traduzido do inglês "instructional" que, literalmente, significa "da instrução" ou "do ensino". Ora, a educação ocorre através do processo de ensino-aprendizagem. Embora nem todo ensino leve à mudança de comportamento num sentido educativo, pode-se dizer que sempre que houver educação há ensino. Do ponto de vista de um sistema, todo ensino deve ser no sentido educativo. Daí poder-se dizer que o professor enquanto ensina está educando. De maneira geral, portanto, pode-se considerar os termos ou expressões "objetivo educacional" e "objetivo instrucional" como significando a mesma coisa, pois quando alguém ensina em nome de uma Instituição, sua intenção é a de educar. Pelo exposto, preferimos o termo "educacional" ou "educativo" (um equivale ao outro) ao invés de "instrucional".

Fundamentando-se na literatura sobre o assunto e, principalmente, tendo em vista a experiência prática, pode-se concluir que a terminologia mais simples e mais funcional deve levar em conta os seguintes conceitos:

a. Objetivo educativo ou educacional é qualquer sentença ou enunciado que expresse um comportamento esperado de um indi- víduo ou grupo após submetê-lo a uma situação de ensino-aprendizagem.

b. Um objetivo educativo pode ser geral ou especifico. Um objetivo geral expressa de maneira global um comportamento. Os comportamentos expressos pelos objetivos gerais implicam processos mais complexos e são atingidos ao final de um período de tempo razoável, difícil de ser determinado. Os comportamentos expressos pelos objetivos especificos devem ser geralmente atingidos a curto prazo ou pelo menos, num espaço de tempo definido. Exemplos de objetivos específicos sāo os comportamentos esperados dos alunos de uma classe após uma sessão de estudo ou após uma unidade de ensino.

c. A operacionalidade de um objetivo refere-se à precisão de sua redação. Quando um objetivo, geral ou especifico, expressa claramente um comportamento, de maneira a não permitir múltiplas interpretações, dizemos que é operacional, ou que está redigido de maneira operacional.

\section{REVISAO DA LITERATURA}

Embora a problemática referente à questão da determinação de objetivos educativos seja ampla e estimulante, restringir-se-á ao problema específico aqui proposto, isto é, o de se estudar em que medida o conhecimento por parte do aluno dos objetivos educativos específicos (e, portanto, operacionalmente redigidos) antes da situação de ensino-aprendizagem, leva-o a um maior rendimento na aprendizagem.

A literatura neste campo é predominantemente prescritiva, fundamentada em princípios gerais de psicologia da aprendizagem. Grande parte das proposições feitas carecem de fundamentação rigorosamente científica e muitas hipóteses estão ainda por ser estudadas.

Encontram-se pontos de vista divergentes em relação à necessidade e possibilidade de se determinar objetivos educativos especificos na fase inicial do planejamento de 
um programa educativo (Oliveira ${ }^{15}, 1973$ ). Para alguns autores, a pré-determinação do comportamento do aprendiz em seus mínimos detalhes levaria a um ensino por demais retalhado, perdendo-se de vista as metas mais gerais da educação. Ausubel e Bruner (apud Oliveira ${ }^{15}$, 1973) afirmam que especialistas en materiais instrucionais se dedicam mais a aspectos triviais e fáceis de serem definidos do que aos aspectos mais fundamentais e que deveriam ser levados em conta na educação.

Outra alegação é a de que tal orientação é anti-democrática, favorecendo um ensino mecânico e desumano que não leva em conta as necessidades e os interesses individuais.

Inexistindo até o presente momento procedimentos sistemáticos que permitam a utilização de técnicas de redação operacional de objetivos e estando a grande maiora dos professores despreparados para esta tarefa, há os que não recomendam sua utilização na prátıca educacional (Pollock ", 1970; Fodor e Dalis 5, 1966).

Em contrapartida, encontram-se inúmeros autores que defendem o ponto de vista contrário, alistando diversos argumentos. Entre eles, podem-se citar:

a. Tendo em vista que, nem sempre o planejador de curriculo é o instrutor, o primeiro deve comunicar ao segundo o que pretende, do contrário o planejador de curso ou o professor não terá elementos para programar a instrução.

b. A redação clara e precisa dos objetivos é condiçāo fundamental para que seja possível selecionar estratégias adequadas à sua consecução e se possa avaliá-los.

Experiências educativas fundamentadas em princípios de tecnologia comportamental têm comprovado consistentemente a eficácia da utilização da redação operacional de objetivos no planejamento educativo. A instrução programada, por exemplo, é uma metodologia de ensino (ujo planejament) inclui, como requisito básico, o uso de objetivos redigidos em termos comportamentais. A elaboração de um texto programado fundamenta-se na aplicação de princípios derivados de diversas ciências. Isto significa imprimir um caráter cientifico ao ensino e à educação que eram até bem pouco considerados uma arte cujo resultado dependia quase que exclusivamente das habilidades pessoais.

Como já se teve oportunidade de mencionar, grande parte dos trabalhos relacionados à questão da determinação de objetivos educativos são de caráter teórico e prescritivo (Bloom ", 1956; Krathwohl 1", 1964; Mager 12, 1961; Pollock 1i;, 1970; Garcia ", 1970; Fodor e Dalis "1966; Popham e Baker 1s, 1970).

Trabalhos desenvolvidos mais recentemente procuraram testar hipóteses relacionadas com alguns aspectos do problema, tentando assim colher evidências empiricas que comprovem uma série de afirmações correntes, mas de valor discutivel. Entre esses trabalhos destacam-se os de Goldberg : (1972), Poole 1; (1970) e Solleder "'" (1972) em que se estuda a fidedignidade da taxonomia de objetivos educativos (dominio cognitivo) proposta por Bloom e col. ${ }^{1}$ (1954). Lewy ${ }^{11}$ (1968) desenvolveu trabatho semelhante em relação ao nivel afetivo. Relaçoes entre objetivos cognitivos e afetivos em termos de aprendizagem foram estudadas por Neidt e Hedlund ${ }^{14}$ (1969). Outras hipóteses relacionadas à questão da determinação de objetivos foram estudadas por Dickinson + (1971), Smith 1: (1970), Poole 1: (1970) e Nagel 1.: (1970).

Em relação ao problema específico que se pretende estudar foi possivel obter apenas um trabalho desenvolvido por Dalis ${ }^{3}$ (1970), no qual o autor se preocupou, especificamente, em verificar o efeito do conhecimento prévio dos objetivos de um programa de educação em saúde, sobre a modificação do comportamento de um grupo de estudantes. Nesse trabalho foram utilizados 143 sujeitos, alunos de 5 classes do curso secundário. Os alunos foram divididos em três grupos. Os do primeiro grupo recebiam informaçóes sobre o que se esperava deles ao final de uma unidade de ensino, através 
dos objetivos educativos redigidos de maneira operacional. Os do segundo grupo recebiam os mesmos objetivos, mas redigidos de maneira imprecisa, possibilitando múltiplas interpretações. Os alunos do terceiro grupo recebiam mensagens que não tinham relação alguma com o conteúdo que seria ensinado.

As conclusões do estudo confirmaram as hipóteses propostas:

a. Os estudantes que tinham recebido antes da aula informação precisa sobre 0 que se esperava deles, apresentaram maior rendimento do que aqueles que tinham recebido como informação objetivos vagos.

b. Os estudantes que tinham recebido antes da aula informação vaga sobre o que era esperado deles, não apresentaram maior rendimento do que aqueles que tinham recebido como informação, objetivos vagos.

c. Os estudantes que tinham recebido objetivos precisos como informação foram mais capazes de selecionar atividades relacionadas aos seus objetivos do que os que receberam como informação objetivos vagos ou sem relação.

\section{H I POTESE}

Por que redigir objetivos educativos de maneira operacional?

Algumas respostas a esta questão já foram dadas anteriormente. Estas respostas, en última análise se resumem na seguinte: porque conduzem a um ensino mais eficiente e a uma maior aprendizagem.

Poderiamos levantar ainda outra questão: além de operacionalizar os objetivos, que outros procedimentos deveria adotar o professor para obter maior rendimento de seus alunos? Nossa resposta seria: informar aos alunos, antes de iniciar o ensino, sobre os comportamentos que são esperados deles após o desenvolvimento do processo. Se esta resposta é verdadeira ou falsa é exatamente o que pretendemos testar.

Nossa hipótese é a seguinte:
"Quando o aluno tem conhecimento prévio dos objetivos educativos visados pelo professor, a aprendizagem é maior do que quando não tem esse conhecimento."

O presente trabalho, da mesma forma que o de Dalis " (1970), foi desenvolvido na área da educação em saúde; não pretende, entretanto, ser uma simples réplica daquele. Foram introduzidas algumas modificaçōes que consideramos relevantes. Entre elas, pode-se citar :

- Equiparação prévia dos grupos estudados em relação a algumas variáveis relevantes para a hipótese em estudo.

- Introdução de controles para evitar a comunicação dos objetivos dos alunos do grupo experimental para os do grupo de controle.

- Verificação da significância da diferença entre médias dos escores não apenas em relação ao grupo como um todo, mas levando-se em conta outros subgrupos dentro do grupo maior.

- A avaliação das mudanças no comportamento não foi realizada apenas em relação à área cognitiva, mas também em relação à afetiva e psicomotora.

\section{METODOLOGIA}

\subsection{Sujeitos}

Foram utilizados 140 alunos de 4 classes da $5^{*}$ série do $1^{\circ}$ Grau de uma escola da rede pública estadual da cidade de São Paulo. Embora a escola e as classes não tenham sido selecionadas por processo estritamente aleatório, não houve critérios prédeterminados para sua escolha.

Os alunos foram divididos em dois grupos que funcionaram como grupo experimental (GE) e grupo controle (GC), respectivamente. Os grupos foram equiparados em relação às variáveis idade, aproveitamento escolar, nível sócio-econômico e ao préteste.

O GE apresentou uma média de idade igual a 11,61 anos e o GC igual a 11,37 
SOSSAI, J. A. - Objetivos operacionais e eficiência da aprendizagem. Rev. Saúde públ., S. Paulo, 11: $157-69,1977$

anos. Aplicado o teste de diferença de médias *, a diferença constatada revelou-se não significativa $(t=1,24)$.

$\mathrm{O}$ aproveitamento escolar foi medido através da média das notas obtidas pelos alunos, em cinco matérias referentes ao primeiro bimestre de 1974. As matérias consideradas foram Português, Matemática, Ciências, Geografia e História. Obteve-se para o GE média das notas igual a 5,79 e para o GC média igual a 5,60. A diferença não $f_{0} i$ significativa $(t=0,82)$.

Para a caracterização sócio-econômica foi utilizada a Escala de Prestígio Social de Hutchinson adaptada por Gouveia 8 (1970). Embora tivessem sido colhidos dados sobre renda familiar e escolaridade do pai, foi considerado satisfatório levar em conta apenas a profissão do pai para determinar () nível sócio-econômico do aluno.

Para maior facilidade, os 7 grupos profissionais da escala citada foram reagrupados em 4 subgrupos assim distribuidos:

Grupos 1 e 2: classe A

Grupos 3 e 4: classe B

Grupos 5 e 6 : classe $C$

(irupo 7 classe D

Verificou-se, através do $\chi^{2}$, que os dois grupos, o $\mathrm{GE}$ e o $\mathrm{GC}$, eram semelhantes em relação ao nível sócio-econômico $\left(x^{2}=6,14\right)$.

As variáveis que se levaram em conta na equiparação dos grupos foram consideradas as de maior relevância para a questão em estudo. Já está suficientemente demonstrada a alta correlação entre cada uma das três variáveis citadas e a capacidade de aprendizagem.

Os resultados do pré-teste vieram confirmar a semelhança entre o GE e o GC. $\mathrm{Na}$ avaliação de conhecimentos, atitudes e práticas, o GE obteve, no pré-teste, média igual a 14,33 pontos e o GC igual a 14,25 pontos. A diferença entre estas médias revelou-se não significativa $(t=0,12)$.

Para efeito de estudo complementar que será discutido posteriormente, foi computada a média de ambos os grupos, levando-se em conta apenas a avaliação dos objetivos cognitivos. $\mathrm{O}$ GE obteve média igual a 7,30 pontos e o GC média igual a 7,11 pontos. A diferença entre elas também se revelou não significativa $(t=0,44)$.

\subsection{Instrumentos * $*$}

Foram elaborados 31 objetivos que especificavam os conhecimentos, as atitudes $\mathrm{e}$ as práticas que os alunos deveriam apresentar após o desenvolvimento de uma unidade de ensino sobre saúde.

Para avaliar a consecução dos objetivos cognitivos foram elaboradas 22 questōes, sendo parte delas constituida de itens de múltipla escolha e parte de perguntas semi-abertas. Nas questões semi-abertas a resposta estava previamente determinada, embora com possibilidade de pequenas variaçōes. A avaliação de atitudes foi feita mediante 6 perguntas e as práticas de saúde através de outras 6 .

As mesmas questões foram utilizadas no pré-teste e no pós-teste. No pré-teste, entretanto, foram apresentadas de uma só vez, ao passo que no pós-teste foram apresentadas em duas etapas, conforme será explicado posteriormente.

Os critérios de avaliação foram expressos através de um "código de correção", a ser utilizado na correção dos testes e na avaliação das respostas aos questionários.

Considerou-se necessário oferecer aos alunos um texto sobre o tema a ser estudado, uma vez que o professor adota um texto como referência básica para suas aulas. O texto foi ilustrado e escrito em linguagem acessivel à criança de $5^{\mathrm{a}}$ série

\footnotetext{
* Foi utilizado o teste $t$ para se verificar se a diferença entre médias foi significativa. Somente para o nível sócio-económico foi utilizado o cálculo da diferença entre os grupos pelos qui quadrado.

** Modelos dos vários tipos de instrumentos usados nesta pesquisa encontram-se à disposição dos interessados, com o autor deste trabalho.
} 
SOSSAI, J. A. - Objetivos operacionais e eficiência da aprendizagem, Rev. Saúde públ., S. Paulo, 11: $157-69,1977$

do 1" Grau, tendo-se o cuidado, entretanto, de manter a forma dos textos convencionais. Cada aluno recebeu uma cópia do texto.

Para a caracterização da amostra foi elaborado um questionário, utilizado para levantamento de dados sobre os alunos. Esse questionário foi respondido pelos alunos com a ajuda dos pais. Foi necessário utilizar este instrumento, pois a escola não dispunha de dados atualizados e completos sobre os alunos.

Foram propostas 21 sugestões de atividades que o professor poderia utilizar no desenvolvimento da unidade de ensino.

\subsection{Procedimentos}

No inicio do primeiro semestre letivo foi solicitado a um professor de ciências que reservasse algumas aulas para o desenvolvimento de uma unidade de ensino sobre saúde, relacionada ao assunto que seria estudado em sua disciplina, como parte de um conjunto de experiências que estavam sencto levadas a efeito, tendo em vista a implantação do Programa de Ensino da Saude na Escola.

Esse professor recebeu treinamento prévio, que consistiu em informaçōes sobre os objetivos da pesquisa, como deveria proceder em relação à metodologia a se. adotada e os instrumentos a serem utilizados. Nossa pretenção era a de testar a hipótese proposta numa situação normal de sala de aula, sem utilizar estratégias diferentes das que são normalmente aplicadas pelo professor de 19 Grau.

As quatro classes que participaram do experimento foram submetidas ao pré-teste para se avaliar conhecimentos, atitudes e práticas já existentes em relação aos objetivos pretendidos. O pré-teste foi aplicado uma semana antes do inicio da pesquisa.

No GC o professor iniciava as aulas abordando diretamente o conteúdo da aula de saúde, ao passo que no $\mathrm{GE}$ os $15 \mathrm{~min}$. iniciais da primeira aula eram reservados para leitura e discussão dos objetivos educativos pretendidos.
A unidade de ensino foi dividida em duas etapas, de acordo com os itens 1 e 2 do texto.

A informação sobre os comportamentos esperados após o desenvolvimento das aulas foi fornecida ao GE por escrito, através dos objetivos educativos. Essa informação foi oferecida em duas etapas de acordo com o desenvolvimento do conteúdo de ensino. $\mathrm{Na}$ folha com os objetivos foram dadas explicações sobre seu significado e sua relação com a avaliação. A folha com os objetivos era colada no caderno de ciências. Este procedimento foi adotado para se evitar que os alunos do GE passassem os objetivos para os do $\mathrm{GC}$.

Da mesma forma, a avaliação foi feita em duas etapas: a primeira abrangeu os objetivos referentes ao tópico 1 do texto e a segunda, os objetivos referentes ao tópico 2.

Nenhuma informação especial foi dada aos alunos sobre os objetivos da pesquisa. Foi-lhes dito apenas que se tratava de uma experiência sobre ensino da saúcle.

Foi gasto um total ce 8 horas/aula para 0 desenvolvimento do programa de educação em saúde, assim distribuidas:

1 h/aula: pré-teste

2 h/aula: desenvolvimento da primeira parte

$1 \mathrm{~h} /$ aula: pós-teste relativo à primeira parte

$3 \mathrm{~h} /$ aula: desenvolvimento da segunda parte

$1 \mathrm{~h} /$ aula: pós-teste relativo à segunda parte

Como se pretendia estudar a hipótese proposta numa situaçāo normal, não se exigiu do professor de classe nenhum planejamento especial de suas aulas. Deveria, apenas, desenvolver as aulas utilizando as mesmas estratégias no $\mathrm{GE}$ e no $\mathrm{GC}$, valendo-se dos instrumentos oferecidos pelo pesquisador. Observaçōes realizadas pelo pesquisador revelaram que as instruções dadas ao professor foram seguidas durante 0 desenvolvimento das aulas. 
SOSSAI, J. A. - Objetivos operacionais e eficiência da aprendizagem. Rev. Saúde públ., S. Paulo, 11: $157-69,1977$

Conforme exposto anteriormente, os objetivos educativos foram avaliados através de prova contendo 33 questões. A cada questão foi atribuído valor 1 , de modo que o total de pontos poderia variar entre zero e 33 .

A contagem dos pontos não foi feita com base semplesmente no resultado do pósteste, mas sim levando-se em conta a diferença entre pontos obtidos no pós-teste $e$ pontos obtidos no pré-teste. A aprendizagem foi calculada pela seguinte fórmula:

$$
\begin{aligned}
\text { em que } \mathrm{A} & =\begin{array}{c}
\text { Aprendizagem } \\
\text { ap }
\end{array} \\
& \begin{array}{l}
\text { pontos obtidos na avaliação } \\
\text { posterior }
\end{array} \\
\text { aa } & =\begin{array}{l}
\text { pontos obtidos na avaliação } \\
\text { anterior }
\end{array}
\end{aligned}
$$

A análise dos resultados obtidos pelos $\mathrm{GE}$ e GC não foi feita levando-se em conta apenas a amostra como um todo. Procurou-se estudar isoladamente os grupos de nivel sócio-econômico alto e baixo, como também us grupos de alunos adiantados e atrasados. $\mathrm{Na}$ constituição destes grupos foram desprezados os que se situavam em posições intermediárias. Assim, os GE e GC de nivel sócio-econômico alto foram constituidos dos alunos classificados no nivel $\mathrm{A}$, isto é, os de classe mais alta. Os grupos de nivel sócio-econômico baixo incluiram os alunos classificados nos níveis $C$ e $D$, sendo desprezados os classificados na classe B.

Para composição dos grupos de alunos adiantados e atrasados, foram adotados os seguintes critérios: os alunos cuja média de notas variava entre 6,0 e 10,0 foram considerados adiantados; os alunos cuja média de notas estava entre zero e 5,0 foram incluidos no grupo dos atrasados. Foram desprezados os que tinham média entre 5,1 e 5,9 .

Tanto para a composição dos grupos quanto ao nível sócio-econômico como em relação ao adiantamento escolar, foram incluídos todos os sujeitos que se enquadravam dentro dos critérios expostos acima.

Deve-se observar que na comparação realizada entre os diversos subgrupos, se aplicou teste de diferença de médias em relação às variáveis levadas em conta na homogeneização do grupo total. Assim, ao se comparar os pontos obtidos pelo GE e GC compostos pelos sujeitos de nível sócioeconômico alto, verificou-se se eram homogêneos em relação à idade e ao adiantamento escolar. O mesmo procedimento foi utilizado ao se comparar os $\mathrm{GE}$ e $\mathrm{GC}$ compostos pelos sujeitos de nivel sócioeconômico baixo, pelos adiantados e pelos atrasados. Em todos os casos observou-se que os grupos eram homogêneos, podendose em princípio atribuir as diferenças na aprendizagem à manipulação da variável independente, isto é, à informação prévia sobre os objetivos educativos.

\section{RESULTADOS}

A comparação entre a média de pontos obtida pelos diversos grupos realizada através do teste $t$, revelou os resultados apresentados na Tabela.

Em vista dos dados apresentados pode-se afirmar que, em princípio, os resultados do presente trabalho não confirmam "in totum" conclusões do experimento desenvolvido por Dalis ${ }^{3}$ (1970), ao qual este muito se assemelha nos seus objetivos e na sua estrutura.

No trabalho de Dalis concluiu-se que "foi possivel aumentar a realização escolar em educação em saúde usando objetivos operacionais antes do ensino, com alunos da escola secundária" (Dalis ${ }^{3}, 1970: 22$ ).

Tomou-se o cuidado de comparar os diferentes grupos em relação à mudança de conhecimentos, atitudes e práticas e em seguida, somente em relação à mudança de conhecimentos, pois este procedimento poderia conduzir a conclusões valiosas do ponto de vista da educação em saúde.

De fato, para o educador de saúde interessa sobretudo conseguir que o individuo modifique práticas inadequadas e adquira novas maneiras de se comportar em relação à saúde. Considerar apenas mudanças nos conhecimentos permitiria a formulação de conclusōes de menor significado. 
SOSSAI, J. A. - Objetivos operacionais e eficiência da aprendizagem. Rev. Saúde públ., S. Paulo, 11: $157-69,1977$

\section{T A B E L A}

Valores de $t$ para o grupo total e subgrupos de acordo com a mudança de comportamento obtida pelo programa educativo

\begin{tabular}{|c|c|c|c|c|c|}
\hline $\begin{array}{l}\text { Areas de } \\
\text { comportamento }\end{array}$ & Grupo Total & $\begin{array}{l}\text { Grupo de } \\
\text { NSE alto }\end{array}$ & $\begin{array}{l}\text { Grupo de } \\
\text { NSE baixo }\end{array}$ & $\begin{array}{c}\text { Grupo alunos } \\
\text { adiantados }\end{array}$ & $\begin{array}{c}\text { Grupo alunos } \\
\text { atrasados }\end{array}$ \\
\hline $\begin{array}{l}\text { Conhecimentos, ati- } \\
\text { tudes e práticas }\end{array}$ & 1,37 & $-0,15$ & $2,2\}^{*}$ & 0,55 & $2,88^{* *}$ \\
\hline Conhecimentos & $2,03^{*}$ & 0,11 & $3,27^{\star *}$ & 0,74 & 1,93 \\
\hline
\end{tabular}

* Diferença significativa ao nível de $5 \%$

** Diferença significativa ao nível de $1 \%$

Sabe-se, por outro lado, que os instrumentos disponiveis para avaliar mudanças de atitudes e práticas, oferecem medidas imprecisas e de pouco valor preditivo (Hays", 1970). Em outras palavras, quando se mede atitudes e práticas através de instrumentos como o questionário (como foi o nosso caso), as respostas podem ser semelhantes no pré e no pós-teste devido à tendência de se responder na direção daquilo que se supōe seja esperado pelo educador.

Era de se esperar, portanto, que houvesse maior diferença entre os diversos grupos experimentais e de controle, quando se levou em conta apenas a aquisição de conhecimentos. Os resultados, entretanto, não foram nessa direção.

Constatou-se que diferenças significicativas e não significativas entre médias ocorreram com igual frequiência, quer levando-se em conta a avaliação das três áreas de comportamento, quer levando-se em conta apenas a avaliação de conhecimentos.

Tomando o grupo como um todo, observou-se uma diferença significativa entre as médias dos $\mathrm{GE}$ e $\mathrm{GC}$ ao nivel de $5 \%$ a favor do GE, quando se levou em conta apenas a aquisição de conhecimentos $(t=2,03)$. Este resultado está de acordo com a proposição feita anteriormente sobre a qualidade da avaliação de conhecimentos em relação à avaliação de atitudes e práticas.
Este resultado, entretanto, é de certa forma contradito quando se toma os alunos mais atrasados. Neste caso, observou-se uma diferença significativa ao nivel de $1 \%$ a favor do GE, quando se levou em conta as três áreas do comportamento $(t=2,88)$, não havendo diferença significativa entre as médias do GE e GC quando se levou em conta apenas conhecimentos.

Fui verificada de maneira consistente diferença significativa a favor do $\mathrm{GE}$ quando se comparou alunos de nivel sócioeconômico baixo. Em relação à avaliação das três áreas do comportamento, foi observada diferença significativa entre as médias dos $\mathrm{GE}$ e $\mathrm{GC}$ ao nível de $5 \%$ $(t=2,23)$ e em relação à avaliação de conhecimentos, a diferença foi significativa ao nível de $1 \%(\mathrm{t}=3,27)$.

Em todos os demais casos, isto é, quando se compararam GE e GC constituidos por alunos de nivel sócio-econômico alto e por alunos adiantados, não se observou diferença significativa entre médias ao nivel mínimo de $5 \%$. Igual resultado foi constatado em relação à avaliação das três áreas tomando-se o grupo como um todo e à avaliação dos conhecimentos dos alunos atrasados.

\section{DISCUSSAO DOS RESULTADOS}

Considerando-se os resultados obtidos de maneira global, observa-se que não há 
consistência entre eles, dando margem a múltiplas hipóteses explicativas desse fenômeno. Entre elas pode-se citar:

a. Terá sido o ensino de tal maneira assistemático que tanto poderia levar como não levar o indivíduo a mudar sell comportamento?

A organização do conteúdo de ensino en sequiências ótimas tem sido enfatizada como um fator fundamental para a aprendizagem (Bruner ", 1971). Metodologias que aplicam de maneira sistemática princípios de tecnologia da educação conduzem geralmente a modificações no comportamento de maneira consistente. E un dos principios fundamentais da tecnologia educacional refere-se à estruturação do conteúdo de insino.

Ém situação normal de sala de aula, , professor não está ainda preocujado com a organização do ensino de maneira a conduzir o aluno por etapas, até as aquisiçōes expressas pelos objetivos educativos.

A utilização de procedimentos issistemáticos no ensino, ou seja, a distribuição mais ou menos casual dos estimulos pode conduzir a um resultado não previsivel ou casual. A mudança de comportan ento pode ser ora no sentido esperado, ora contrário.

b. Uma condição fundamental para que os objetivos educativos tenhan alguma influência sobre a aprendizagem is que eles sejam compreendidos pelos alunos. Provavelmente, quanto mais elevado o nivel de escolaridade de um grupo, maior será a possibilidade de compreensão dos objetivos de um programa educativo.

No presente caso, será que us alunos da $5^{a}$ série do 10 Grau foram cápazes de compreender os objetivos propostos? Somente numa pesquisa mais ampla se poderia testar esta hipótese.

c. Será que os procedimentos utilizados pelo professor para explicar os objetivos foram adequados? Diversas formas de apresentação dos objetivos poderiam ser testadas, seguidas de verificação sobre a compreensão dos mesmos. Em no:sso trabalho prático temos observado que a propo- sição de questões sobre determinado tema, para serem respondidas pelos alunos, podem funcionar de maneira semelhante à informação sobre os objetivos, tal como foi utilizada no presente trabalho. De fato, um objetivo redigido de maneira operacional assemelha-se muito a uma pergunta ou questão tal como é proposta, por exemplo, quando se utiliza a técnica do estudo dirigido.

d. A influência do (onhecimento prévio) dos objetivos pode variar em função do conteúdo educativo ou do ensino e da metodologia. Como foi assinalado no inicio deste trabalho, certos métodos de ensino, como a instrução programada, fundamentam-se en principios de tecnologia da educação que facilitam grandemente a aprendizagem, podendo anular o efeito de outros procedimentos.

Diferentes conteudos de ensino podem ter impacto diferente sobre o educando. $\mathrm{Na}$ época presente, mudar comportamentos de individuos tendo em vista economia de gasolina, por exemplo, torna-se muito mais fácil do que ensinar regra de très. A motivação en relação a determinado tema pode ser tão relevante, que outros recursos metodológicos, como aquele utilizado no presente trabalho, têm pouca ou nenhuma influência sobre a aprendizagem.

e. Houve maior consistência nas diferenças entre GE e GC quando se levou em conta alunos de nivel sócio-econômico baixo) e alunos atrasados. Em relação aos alunos de nível sócio-econômico baixo, houve diferença significativa entre $\mathrm{GE}$ e $\mathrm{GC}$ tanto quando se levou em conta as três áreas do comportamento $(t=2,23)$ como quando se computou apenas a aquisição de conhecimentos $(t=3,27)$. Em relação ao grupo dos atrasados, houve diferença significativa quando se levou em conta as três áreas de comportamento $(t=2,88)$.

Pode-se supor que o conhecimento dos objetivos educativos pode ser relevante para alunos de nível sócio-econômico baixo $\mathrm{e}$ para os alunos mais atrasados, porque os objetivos representam um auxilio impor- 
tante para estes grupos. Sabe-se que os alunos mais atrasados e de nivel sócio-econômico mais baixo são os que mais necessitam de ajuda extra para conseguirem melhorar sell rendimento. No caso, os objetivos podem ter se constituid() nesse auxílio) extra.

f. Uma última hipótese poderia ser proposta, isto é, a de que a informação aos alunos sobre os objetivos pretendidos, antes de submetê-los à situação de ensino-aprendizagem, é um procedimento irrelevante, não influenciando significativamente a aprendizagem.

Novas pesquisas abordando diferentes aspectos são fundamentais para () estabelecimento de conclusões mais definitivas sobre () assunto em questão e poderão fornecer principios para aplicação sistemática a programas educativos.

\section{CONSIDERAÇOESS FINAIS}

A relevância da questão em estudo é obvia, tendo en vista que todas as pesquisas sobre aprendizagem têm como finalidade, en última análise, conseguir maior eficiência no processo de modificação do comportamento dos indivíduos.

Os principios cientificos subjacentes a uma teoria do ensino ou da aprendizagem vão se traduzir na prática em procedimentos que, se utilizados pelo professor, conduzirão a maior eficiência no processo de ensinoaprendizagem.

Muitas proposições têm sido feitas e continuam a ser feitas a respeito das estratégias que devem ser utilizadas para se conseguir, de maneira eficiente, que os individuos adquiram novos conhecimentos, mudem suas atitudes e adotem práticas que thes proporcionem maior ajustamento ao meio em que vivem. Grande número delas têm sido propostas pelo que se denomina tecnologia da educação ou tecnologia do ensino. Muitas destas proposições, entretanto, implicam na utilização de meios sofisticados de ensino, e que geralmente não são acessíveis ao professor comum. Esta situação se torna mais grave quando se verifica que há uma distorção a respeito daquilo que constitui tecnologia de educação, confundindo-se esta com recursos audiovisuais de ensino como rádio, cinema, televisão e o próprio ensino por computador.

Considerando a tecnologia da educação na sua verdadeira acepção, isto é, como aplicação sistemática de princípios científicos no processo de modificação do comportamento, cabe ao pesquisador oferecer instrumentos acessiveis e práticos, que podem ser utilizados com facilidade pelo professor comum, sem ônus especial para ele ou para os sistemas de ensino.

Neste sentido, a questão) a que nos propusemos estudar representa un aspecto altamente significativo no campo da pesquisa educacional aplicada, pois as possiveis conclusōes derivadas desta experiência têm en vista oferecer ao educador instrumentos de trabalho acessiveis e ao mesmo tempo de grande significado prático.

Dadas as limitações da experiência por nós realizada, as conclusões serão também limitadas, o que não deixa de significar alguma contribuição dentro do (ampo da pesquisa educacional.

No caso especifico do nosso estudo, não houve uma tendência uniforme e coerente, como já ficou demonstrado. Não se pode, portanto, afirmar que ficou comprovado ser verdadeira a hipótese proposta inicialmente. Nem tampouco pode-se negar a hipótese com base nos dados obtidos.

Embora de grande relevância teórica $\mathrm{e}$ prática a hipótese estudada no presente trabalho, para a obtenção de conclusões mais significativas, é necessário o desenvolvimento de pesquisas mais amplas com o objetivo de testar outros aspectos relacionados ao problema em questão. Para isto, os seguintes aspectos devem ser considerados:

a. Deve-se trabalhar com maior número de sujeitos.

b. As amostras devem ser mais representativas dos diversos estratos sociais. 
SOSSAI, J. A. - Objetiros operacionais e eficiência da aprendizagem, Rer. Saúde públ., S, Paulo, 11:157-69, 1977

c. Deven ser realizados experimentos com sujeitos dos diferentes graus escolares.

d. Diversos conteudos educativos ou de ensino devem ser utilizados.

e. Diferentes metodologias deven ser empregadas, não se excluindo a instrução programada.

f. Mudanças na área cognitiva, afetiva e psicomotora devem ser consideradas em conjunto e separadamente.

g. Devem ser desenvolvidos experimentos não só com professures regentes de classe, mas tambén com outros profissionais especialmente treinados e com experiência em pesquisa.

\section{CONCLUSOES}

A partir dos dados obtidos no presente trabalho, pode-se formular as seguintes conclusões:

a. Não se pode afirmar que é possivel aumentar sistematicamente o rendimento escolar de alunos de $5^{4}$ série do 1" Grau no ensino da saude, informando-os previamente sobre os objetivos educativos pretendidos pelo professor. b. A informação prévia aos alunos sobre us objetivos do ensino levou a um aumento significativo na aprendizagem, quando se trabalhou com grupos de nivel sócio-econômico baixo. Quando se considerou o grupo estudado como un todo, houve aumento significativo na aprendizagem quando se levou en conta apenas a aquisiçăo de conhecimentos. Constatou-se tambén um aumento significativo na aprendizagem quando se trabalhou com us alunos mais atrasados. Neste caso, porém, o aumento só foi constatado yuando se avaliou as três áreas de comportamento conjuntamente Nos demais casos, isto é. quando se levou em conta mudanças de comportamento nas três áreas, do grupo como um todo, do grupo de nivel sócio-econômico alto e do grupo dos adiantados, como também quando se avaliou apenas ayuisição de conhecimentos do grupo de nivel sócio-económico alto, do grupo dos adiantados e dos atrasados, não se observou aumento significativo na aprendizagem.

c. Para a obtenção de conclusões mais definitivas, recomenda-se a realização de pesquisas mais amplas, em que se possa testar hipóteses complementares relacionadas à questão analisada no presente trabalho.

Sossal, J. A. - [Operational objectives and effectiveness of learning. Rev. Saúde públ., S. Paulo, 11:157-69, 1977.

ABSTRACT: The purpose of this stud! is to provide data on whether student achievement can be influenced significantly by providing students, before instruction, information on what is expected of them as an outcome of instruction. The Ss were selected from four fifth-grade classes of the elementary course. Of the $140 \mathrm{Ss}, 69$ from two classes were assigned to experimental group (EG) and 71 from two other classes were assigned to the control group ( $C G$ ). The $S S$ of $E G$ received precisely stated behavioral objectives while SS of $C G$ didn't receive any. information about objectives whatsoever. $E G$ and $C G$ as a whole, high and low socio-economic $E G$ and $C G$, high and low grades $E G$ and $C G$ were compared taking into account changes in knowledge, attitudes and practices on the one hand, and changes only in knowledge on the other hand. Although EGs consistently showed grater achievement than $C G$ s, the results were not conclusive. Significant differences in achievement were observed at random when different groups were considered.

UNITERMS: Teaching, educational objectives. Teaching, operational objectives. Learning. 


\section{REFERENCIAS BIBLIOGRÁFICAS}

1. BLOOM, B. S., ed. Taxonomy of educational objectives, handbook I: Cognitive domain. New York, David McKay, 1956.

2. BRUNER, J. S. o processo da educacão. São Paulo. Ed. Nacional, 1971.

3. DALIS, G. T. Effects of precise objectives upon student achievement in health education. J. exp. Educ., 39:20-3, 1970.

4. DINCKINSON, D. J. School nursing becomes accountable in education throuth behavioral objectives. J. Sch. Hlth., 41 : 433-7, 1971 .

5. FODOR, J. T. \& DALIS, G. T. Health instruction-theory and application. Philadelphia. Lea \& Febiger, 1966.

6. GARCIA, A. L. O. La redacción de objetivos en terminos de conducta observable. San Juan de Puerto Rico, Universidad de Puerto Rico, Facultad de Pedagogia, 1970.

7. GOLDBERG, M. A. A. Uma análise de fidedignidade da taxonomia de objetivos educacionais de Benjamin Bloom. Cad. Pesq., Fund, Carlos Chagas, (6): 56-71, 1972.

8. GOUVEIA, A. J. Professores de amanhã: um Estudo de Escolha ocupacional. São Paulo. Liv. Pioneira Ed,, 1970.

9. HAYS, W. L. Quantificação em psicologia. São Paulo. Ed, Herder, 1970.

10. KRATHWOHL, D. R. et al. Taxonomy of educational objectives, handbook II: Affective domain. New York, David McKay, 1964
11. LEWY, A. The empirical validity of major properties of a taxonomy of affective educational objectives. $J$. exp. Eauc., $36: 70-7,1968$.

12. MAGER, R. F. Preparing objectives for programmed instruction. San Francisco, Fearon Public., 1961.

13. NAGEL, C. A behavioral objectives approach to health instruction. J. Sch. Hith., 40:255-8, 1970.

14. NEIDT, C. O. \& HEDLUND, D. E. Longitudinal relationship between cognitive and affective learning outcomes. J. exp. Educ, 37:56-60, 1969.

15. OLIVEIRA, J. B. A. Tecnologia educacional: teorias da instrugão. Petrópolis, Ed, Vozes, 1973.

16. POLLOCK, M. B. Behavioral objectives: a process approach to health education. Int. J. Hith. Educ., 13:27-35, 1970.

17. POOLE, R. L. Characteristics of the taxonomy of educational objectives: cognitive domain. Psych. in the Sch. (4): 379-85, 1970.

18. POPHAM, W. J. \& BAKER, E. L. Planning an instructional sequence. Englewood Cliffs, N. J., Prentice-Hall, 1970.

19. SMITH, I. L. IQ, creativity, and taxonomy of educational objectives: cognitive domain. J. exp. Educ., 38:58-60, 1970.

20. SOLLEDER, M. K. Evaluation in the cognitive domain. J. Sch. Flth., 42: 16-20, 1972.

Recebido para publicasão em 08/03/1976. Aprovado para publicasão em 17/12/1976. 